\title{
Goggle Mouse Controlled For Handicapped and Paralyzed People
}

\author{
Sheikh Rafik Manihar ${ }^{1}$, Nalin Tiwari ${ }^{2}$, Jayant Rajpurohit ${ }^{3}$ \\ ${ }^{1,2}$ Electronics and Instrumentation, Chhatrapati Shivaji Institutes of Technology, Durg, Chhatisgarh, India. \\ ${ }^{3}$ Electronics and telecommunication, Assistance teaching, Chhatrapati Shivaji Institutes of Technology, Durg, \\ and Chhattisgarh, India.
}

\begin{abstract}
The basic idea of the projects is to build goggle by using human machine interface which can be used to control mouse using head-tilt and eye-blink. This mouse-emulating device can be found to be most useful by physically handicapped people who can no longer control the computers using their hands. Since the device relies on user's head and eye movement, it can be used even by patient who are paralyzed from shoulder downward. Simple head movement doesn't require too much energy and neither does eye blinking. Therefore user won't get tired from using this device. In this device or goggle we are using $2 D$-accelerometer for detecting the movement of the head according to this, the movement of the cursor has been done. A photo sensor detects eye blinking. The Infrared transceiver consists of a $935 \mathrm{~nm}$ IR transmitter and a phototransistor mounted on the same unit. This detects a strong increase in the reflected signal upon intentional long blink as compared to normal eye blink. Artificial hands and legs have been proven to be quite effective and satisfactory for a limited number of activities. Now-a-days, a computer is a common thing at home. The failure of these artificial limbs in enabling the amputees to successfully handle a computer is evident since one can exercise a limited control on the mechanical limbs attached. Moreover many amputees have a job and work in offices wherein they have to use a computer. Hence device should be thought of which would allow easy and smooth control over computer.
\end{abstract}

Keywords - IR Transceiver, Handicapped, Mouse, Goggle, Accelerometer, Micro-Controller.

\section{INTRODUCTION}

To develop a human machine interface emulating function of a mouse for disabled or paralyzed persons. Since the device relies on user's head and eye movements, it can be used even by the patient who is paralyzed from shoulder downward. Simple head movement doesn't require too much energy, and neither does eye blinking. Therefore user won't get tired from using this device. We use accelerometer to detect the movement. When the head of the user is tilted up/down or left/right, the reading from the accelerometer is subtracted from the value of a pre-define reference point. The difference determines the level of head tilt. A photo sensor detects eye blinking. The Infrared transceiver consists of a $935 \mathrm{~nm}$ IR transmitter and a phototransistor mounted on the same unit. This detects a strong increase in the reflected signal upon intentional long blink as compared to normal eye blink. The output of both the sensor are given to the ADC input and after on the microcontroller. After the signals are interpreted by the microcontroller, mouse instructions are sent to the computers. The processed digital information is transmitted to the PC through the serial port. Since many years, various Artificial Limbs and services are offered worldwide to people who have permanent or long term impairment. Artificial hands and legs have been proven to be quite effective and satisfactory for a limited number of activities. Now-a-days, a computer is a common thing at home. The failure of these artificial limbs in enabling the amputees to successfully handle a computer is evident since one can exercise a limited control on the mechanical limbs attached. Moreover many amputees have a job and work in offices wherein they have to use a computer. Hence device should be thought of which would allow easy and smooth control over computer. Thus every growing need of the computer is paralleled by ever increasing ways to work with them.

\section{Hardware Description Blink Detection Circuit}

For the input mechanism, in order to detect the blink of the eye, we needed to have a photo sensor which acts as a transceiver. Initially we selected OPB710, a reflective object sensor manufactured by optek, for the purpose. However, as the particular model was not available, we settled for OPB706 which has similar characteristics to the former, only a shorter range. The OPB706 consists of an infrared light emitting diode and an NPN silicon phototransistor mounted "side-by-side" on parallel axes in a black plastic housing. On OPB706 the LED and phototransistor are molded using dark infrared transmissive plastic to reduce ambient light noise. 
The phototransistor responds to light from the emitter when a reflective object passes within its field of view of the device.

Led Peak Wavelength $=935 \mathrm{~nm}$.

Reflection distance $=0.050 "(1.27 \mathrm{~mm})$.

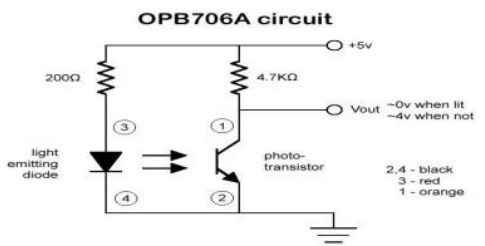

Fig 2: Circuit Diagram of Sensor.

Head Tilt Sensor (Accelerometer) MMA7260Q

We decided to use an accelerometer for the determination of the degree of user's head tilt. Accelerometer uses the force of gravity as input vector to determine orientation of object in space. When oriented parallel to earth's surface it can be used to detect the relative tilt of head. One of the most popular applications of the MMA7260Q is tilt measurement. The accelerometer uses the force of gravity as an input vector to determine orientation of an object in space. An accelerometer is most sensitive to tilt when its sensitive axis is perpendicular to the force of gravity, i.e. parallel to the earth's surface. At this orientation its sensitivity to changes in tilt is highest.

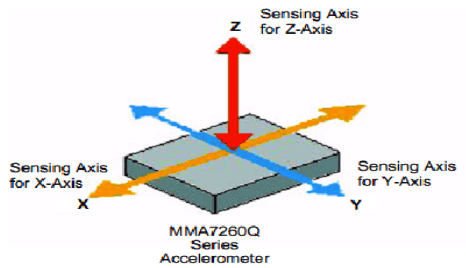

The accelerometer consist of a g-cell which does the work of sensing change in acceleration and hence the tilt. G-cell consists of two capacitive sensing cells, formed using 3 beams, where the middle beam is movable. Thus, these are 2 back-back capacitors. Now, we know that capacitance is given as:

\section{$\mathbf{C}=\mathbf{\varepsilon A} / \mathbf{d}$}

Where, $\mathrm{A}$ is the area of the beam.

$\mathcal{E}$ is the dielectric constant.

$\mathrm{D}$ is the distance between the beams.

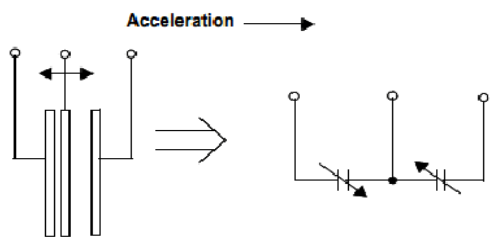

Fig 3: Simplified Transducer Model of G-Cell.

So, whenever there is a tilt in the either $\mathrm{X}$ or $\mathrm{Y}$ axis, the middle beam moves changing its distance with respect to the other two fixed plate. This changes the resultant capacitance of the G-cell. This change in capacitance is used to determine the output of the accelerometer. The IC also signal conditions and filters the output signal, providing a high level output voltage that is ratio-metric and proportional to acceleration.

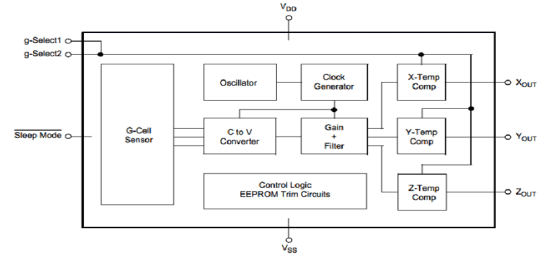

Fig 3: Functional Block Diagram of Accelerometer.

\section{A/D converter}

The output of the sensor is fed into the A/D converter for the conversion of analog signal which is the output of the sensor to digital signal which is fed to the microcontroller for further processing of data. The reason why we are using A/D converter is that microcontroller works on the digital signal for the further processing of data. Here we are using HD74LS90P to provide necessary clock pulse to the ADC. 


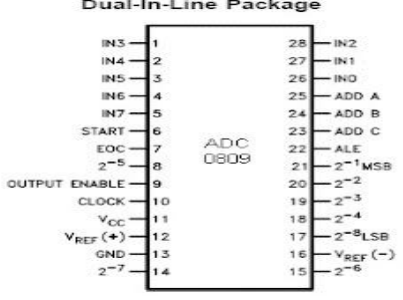

Fig 3: Pin Diagram of ADC.

\section{Micro- controller}

The output of IR transceiver and accelerometer are detected by micro-controller after getting processed through A/D convertor. The function of the micro-controller is to receive the data from A/D converter, process it and then send suitable signals to the computer's serial port. The AT89S52 is a low-power, high-performance CMOS 8-Bit microcontroller with $8 \mathrm{k}$ bytes of in-system programmable Flash memory. The device is manufactured using Atmel's high-density non volatile memory technology and is compatible with the industrystandard 80C51 instruction set and pin out. The on-chip Flash allows the program memory to be reprogrammed in-system or by a conventional non volatile memory programmer. By combing a versatile 8-bit CPU with insystem programmable Flash on a monolithic chip, the Atmel AT89S52 is a powerful microcontroller which provides a highly-flexible and cost-effective solution to many embedded control applications.

\section{RS-232 Interface (MAX 232)}

RS-232 is a serial communication cable which is needed for communication between the computer and the micro-controller. The RS-232 communication is preferred because;

1) It requires only 2 lines and a common ground.

2) Serial communication is easy to implement.

The micro-controller circuitry works with TTL $\operatorname{logic}+5 \mathrm{~V}$ as logic 1 and $0 \mathrm{v}$ as logic 0 . However RS232 standard have different voltage levels. They are $3-15 \mathrm{~V}$ for TTL logics 0 and -3 to $-15 \mathrm{~V}$ for TTL logic 1.Hence conversion of TTL levels to these levels are required. This is done by MAX 232 IC with the help of the $1 \mu \mathrm{f}$ capacitor connected to it. The output of MAX232 is given to the RS-232 connector. It is then given to the PC through the DB-9 connector.

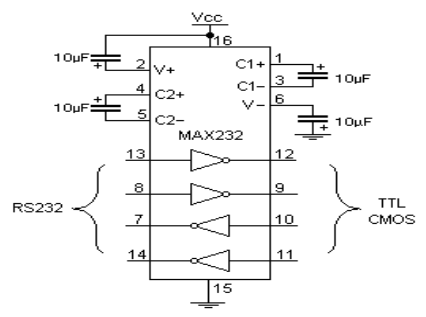

\section{Power supply}

Fig 3: Pin Diagram of MAX 232.

A fixed voltage power supply producing constant $+5 \mathrm{~V}$ consists of step down transformer, a bridge rectifier, filter capacitors C1 and 3 terminal regulator IC LM7805. A step down transformer is selected in such a way that it produces $9 \mathrm{~V}$ at the input of IC. This power supply is capable of supplying $+5 \mathrm{v}$ and load current up to $500 \mathrm{~m} \mathrm{~A}$. The capacitor C2 connected between output terminal and ground cancels out any inductive effect due to long distribution leads. Input capacitor $\mathrm{C} 1$ is used to improve transient response of the regulator IC, i.e. response of regulator to sudden changes in load. It is also helpful in reducing the noise present in the output. Dropout voltage (Vin-Vout) needs to be at least $2 \mathrm{~V}$ under all operating conditions for proper operation of regulator.

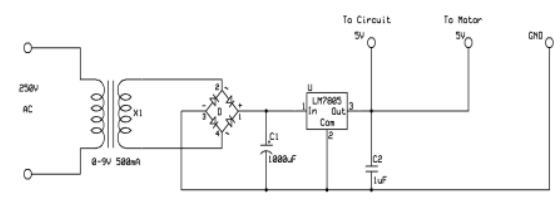

III.

Fig 3: Circuit Diagram of Power Supply.

We are use two software's for the working of our projects. One code is placed in microcontroller for transmission of information regarding and click. Another program runs on PC to collect the data from serial port and set mouse cursor accordingly. We use the followings software's: 
1) Keil C software- To write program for 8052.

2) Visual basic 6.0-To receive data at PC.

3) Hyperlink terminal-To show input on PC.

During the program, we first check the clicking condition that is, whether the output from IR receiver is more than $40 \mathrm{mV}$ as compared to its initial value.

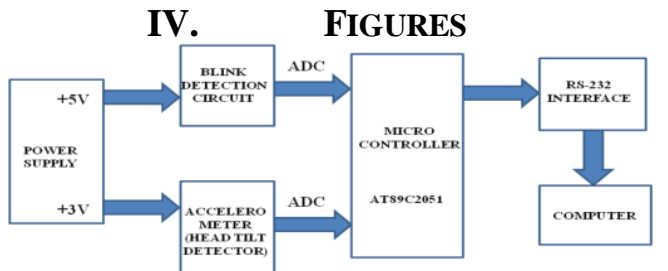

Fig 1: Block Diagram.

V. RESULT

\section{Blinking Testing}

Table 1. Testing of Blinking Detector.

\begin{tabular}{|c|c|c|}
\hline Parameter & $\begin{array}{c}\text { Value in } \\
\text { dark Condition }\end{array}$ & Value in Brighter Condition \\
\hline $\begin{array}{l}\text { IR Receiver } \\
\text { Voltage for open } \\
\text { eye }\end{array}$ & $\begin{array}{ll}\mathrm{mV} & 510\end{array}$ & $535 \mathrm{mV}$ \\
\hline $\begin{array}{lr}\text { IR } & \text { Receiver } \\
\text { Voltage } & \text { for } \\
\text { closed eye } & \end{array}$ & $\begin{array}{ll} & 560 \\
\mathrm{mV} & \end{array}$ & $565 \mathrm{mV}$ \\
\hline $\begin{array}{l}\text { Difference in } \\
\text { Voltage }\end{array}$ & $\mathrm{mV}$ & $30 \mathrm{mV}$ \\
\hline $\begin{array}{l}\text { Decided } \\
\text { Threshold value }\end{array}$ & $40 \mathrm{mV}$ & $40 \mathrm{~V}$ \\
\hline
\end{tabular}

\section{Accelerometer Testing}

Table 2. Testing of Accelerometer

\begin{tabular}{|c|c|c|}
\hline Position & X Output & Y Output \\
\hline Initial & 1.79 & 1.79 \\
\hline $45^{\circ}$ to the Right & 1.91 & 1.79 \\
\hline $90^{\circ}$ to the Right & 2.01 & 1.79 \\
\hline $45^{\circ}$ to the Left & 1.67 & 1.79 \\
\hline $90^{\circ}$ to the Left & 1.57 & 1.79 \\
\hline 45 Upwards & 1.79 & 1.98 \\
\hline 90 Upwards & 1.79 & 2.08 \\
\hline 45 Downwards & 1.79 & 1.74 \\
\hline 90 Downwards & 1.79 & 1.64 \\
\hline
\end{tabular}

\section{REFERENCES}

[1] Thomas B. Moeslund Amd Lau Norgaard, 2003, "A Brief Overview of Hand Gesture Used In Wearable Human Computer Interface”, Technical Report: CVMT03-02, ISSN 1601-3646, Laboratory of Computer Vision and Media Technology, Aalborg University, Denmark.

[2] L.M. Roylance and J.B Angell, “A Batch Fabricated Silicon Accelerometer,” IEEE Trans. Electron Devices, Vol. ED-26, Pp.19111917.

[3] Adam J.Sporka, Sri H. Kurnaiwan, Pavel Slavik, Acoustic Control of Mouse Pointer, Information Society, 2006, Vol.4, Num.3, Pp.125-131.

[4] A7 Engineering. Embeddedblue 500 User Manual, Revision E, April6, 2005.

[5] Amy K. Karlson, Benjamin B.Bederson, John Sangiovanni, Applens And Launchtile; Two Design For One Handed Thumb Use On Small Devices, Preceedings Of The SIGCHI Conference On Human Factors In Computing System, April 02-07, 2005,Portland, Oregon,USA.

[6] J.S. Chae,H. Kulah, K. Najafi, “A High Sensitivity Silicon On-Glass Lateral Microaccelerometer.” Nanospace’ 2000, Houston, TX.

[7] R. Dillmann, "Teaching And Learning Of Robot Tasks Via Observation Of Human Performance," In Robotics And Autonomous System, Vol.47, No.2-3, PP.109-116. 